\title{
Review: advice from doctors and nurses, behavioural interventions, nicotine replacement treatment, and several pharmacological treatments increase smoking cessation rates
}

\author{
Lancaster T, Stead L, Silagy C, et al, for the Cochrane Tobacco Addiction Review Group. Effectiveness of interventions to help \\ people stop smoking: findings from the Cochrane Library. BMJ 2000 Aug 5;321:355-8.
}

\section{QUESTION: Are smoking cessation interventions effective?}

\section{Data sources}

Reviews were identified by searching the Cochrane Library.

\section{Study selection}

Reviews were selected if they included randomised contobacco use that had $\geqslant 6$ months of follow up with outcomes of sustained abstinence or point prevalence stop rates.

\section{Data extraction}

Extracted data included interventions and outcomes.

\section{Main results}

20 systematic reviews were available in the Cochrane Library. 1 review (including 31 trials and $>26000$ participants who were smokers) examined simple advice given by doctors during routine care and showed that the intervention increased quit rates (weighted odds ratio [OR] 1.7, 95\% CI 1.5 to 2.0). Another review of individual counselling given by nurses also showed increased stopping rates.

Behavioural interventions for smoking cessation, in the forms of individual counselling (1 review) or group therapy (1 review), showed increased stopping rates. The review of individual counselling (9 trials) showed that it was more effective than brief advice or usual care (weighted OR 1.6, CI 1.3 to 1.9). Group therapy was more effective than self help materials, but was not more effective than interventions involving personal contact. 1 review of aversion therapy ( 24 trials), in which the pleasurable stimulus of smoking was paired with an unpleasant stimulus, found little evidence of beneficial effect. 1 review showed that self help interventions had no additional benefit over brief personal advice, but were more effective than no intervention (weighted OR 1.2, CI 1.0 to 1.5). A comparison of self help interventions (8 trials) showed that personalised materials were more effective than standard materials (weighted OR 1.4, CI 1.1 to 1.8 ), and that some benefit could also be provided by telephone support (7 trials).

A review of nicotine replacement treatment $(>90$ trials) showed that all forms of this treatment were effective (weighted OR 1.7, CI 1.6 to 1.8). A review of anxiolytics and antidepressants showed that anxiolytics were not effective but that the antidepressants, bupropion and nortriptyline increased stopping rates. A review of the antihypertensive, clonidine (6 trials), showed effectiveness (weighted OR 1.9, CI 1.3 to 2.7) but its usefulness was limited by its side effect profile. A review of lobeline showed no consistent effect of trolled trials of interventions to reduce or prevent

treatment at 6 weeks. Reviews of acupuncture, hypnotherapy, and exercise showed limited evidence of effectiveness for smoking cessation.

\section{Conclusion}

Counselling by doctors and nurses, behavioural interventions (individual counselling or group therapy), nicotine replacement treatment, and several pharmacological interventions (such as the antidepressants, bupropion and nortriptyline) increase smoking cessation rates.

\section{COMMENTARY}

Lancaster $e t$ al have done a credible overview of smoking cessation interventions, making this valuable information in the Cochrane Library accessible to a broader audience. The authors have summarised the results of 20 relevant, methodologically rigorous, systematic reviews from the Cochrane Database of Systematic Reviews. This electronic database, available on the internet and on CD ROM, contains hundreds of systematic reviews produced and regularly updated by a dedicated cadre of expert volunteers. Abstracts of each of the 20 source reviews included in this overview can be accessed free of charge at (http:// www.update-software.com). The findings of this review will not be news to many people who are generally familiar with research on smoking cessation. For the most part, the conclusions mirror the recommendations of the recently updated US Public Health Service's (USPHS) Clinical Practice Guideline on Treating Tobacco Use and Dependence. ${ }^{1}$ It is reassuring that, despite the use of somewhat different metaanalytic and interpretive methods, both reports agree that (1) all forms of nicotine replacement appear to be about equally effective, (2) bupropion sustained release is efficacious, (3) all forms of counselling are effective, (4) self help materials are of limited effectiveness, and (5) anxiolytics are ineffective. The USPHS report is more supportive of aversive smoking techniques than is the Cochrane review. The Cochrane review interprets the evidence as favouring individually tailored self help material over standard ones, while the USPHS report calls for their further study. The USPHS interprets the evidence on combining 2 different forms of nicotine replacement treatment favourably and recommends this approach, while the Cochrane review is more cautious and calls for further study. Both the USPHS report and the 20 individual Cochrane reviews are much richer in detail than this overview and are highly recommended for those with a serious interest in smoking cessation.

Roger Luckmann, MD, MPH University of Massachusetts Medical Center Worcester, Massachusetts, USA

this abstract appears in

Evidence-Based

Nursing,

Evidence-Based

Medicine, and ACP

Journal Club.
1 US Public Health Service. Treating tobacco use and dependence: Summary. Rockville, MD: US Public Health Service, June 2000. http://www.surgeongeneral.gov/tobacco/. 